\title{
Rectal Small Cell Carcinoma:
} A Case Report and Review of the Literature

\author{
Paulina Spiliopoulou Udaiveer Panwar $^{\mathrm{b}}$ \\ Neville Davidson ${ }^{b}$ \\ aGuy's and St Thomas' NHS Foundation Trust, London, and 'Broomfield Hospital, \\ Mid Essex Hospital NHS Services, Chelmsford, UK
}

\section{Key Words}

Extrapulmonary small cell carcinoma $\cdot$ Rectal carcinoma $\cdot$ Chemotherapy $\cdot$ Radiotherapy

\begin{abstract}
We present a rare case of small cell carcinoma of the rectum presenting with rectal bleeding and discomfort in a fit 51-year-old gentleman. Our patient was treated with a combination of chemotherapy and radiotherapy and remains alive and free of disease 6 years after diagnosis. Our patient experience and review of the literature is presented highlighting the uncertainties regarding the biological behaviour and management.
\end{abstract}

\section{Introduction}

Small cell carcinomas (SCC) are malignancies deriving from neuroendocrine tissue and although the gastrointestinal tract contains the largest population of neuroendocrine cells, these malignancies are rare. Through amine precursor uptake and decarboxylation system, neuroendocrine cells are capable of synthetizing, storing and secreting a variety of neuroamines, neuropeptides and related substances [1].

Small cell neoplasia most commonly originates in the lung but also other sites such as skin, thymus, kidney, breast, ovary, uterus, urinary bladder, hepatobiliary tree, pancreas, and salivary glands [2] (extrapulmonary small cell carcinoma, EPSCC). More specifically, the colorectal SCC accounts for $0.2 \%$ [3] to $1.5 \%$ [4] of all colonic cancers and is considered to be an aggressive neoplasm with rapid growth and early spread carrying poor prognosis [3]. The chemosensitivity of the EPSCC is reported to be similar to small cell lung cancer (SCLC) with similar chemotherapeutic agents. 


\section{Case Report}

A 51-year-old fit gentleman presented with a short history of pelvic discomfort, perianal pain and bleeding per rectum. The patient was non-smoker and the only comorbidity was hypertension-induced cardiomegaly. Per-rectal examination confirmed a large obstructing tumour in the lower rectum. Imaging (CT chest/abdomen/pelvis; fig. 1) confirmed a large rectal mass extending from the anorectal junction to the mid rectum with multiple enlarged perirectal and mesorectal lymph nodes.

The rectal biopsy demonstrated a poorly differentiated malignant tumour with surface ulceration (fig. 2). Immunohistochemistry revealed that tumour cells were negative for cytokeratin, LCA (lymphoid marker) and S100 but positive for CD56 (neural cell adhesion molecule, NCAM) and chromogranin, consistent with a diagnosis of small cell (neuroendocrine) carcinoma (fig. 3 ). Staging CT scan and bone scan excluded any metastatic spread beyond the pelvis.

The case was discussed in the colorectal multidisciplinary meeting and was felt to be inoperable at the time and the recommendation was to treat with a combination of chemotherapy and radiotherapy. The patient was commenced on chemotherapy (August 2005) consisting of alternate cycles of cyclophosphamide $800 \mathrm{mg} / \mathrm{m}^{2}$ day $1+$ liposomal doxorubicin $50 \mathrm{mg} / \mathrm{m}^{2}$ day $1+$ vincristine $1.4 \mathrm{mg} / \mathrm{m}^{2}$ day 1 (CAV) and carboplatin AUC 5 day $1(600 \mathrm{mg})+$ etoposide $100 \mathrm{mg} / \mathrm{m}^{2}$ i.v. day 1 , day 2 and day 3 (EP) repeated every 3 weeks for 6 cycles. Liposomal doxorubicin was chosen as the patient had preexisting hypertension-induced cardiomegaly. The patient tolerated chemotherapy well and his symptoms improved drastically following chemotherapy.

Following completion of chemotherapy, the patient received consolidation radiotherapy to the pelvis to a dose of $30 \mathrm{~Gy} / 10 \#$. A restaging CT scan of chest/abdomen/pelvis in January 2006 demonstrated good partial response with minimal residual rectal disease (thickening) and no distant metastasis. Maintenance chemotherapy was offered with weekly i.v. etoposide $\left(100 \mathrm{mg} / \mathrm{m}^{2}\right) 3$ weeks on and 1 week off for further 6 cycles.

A repeat $\mathrm{CT}$ at this stage confirmed unchanged appearance of rectal wall thickening and no new


repeated at 4 weekly intervals. Subsequent CT scan (December 2006) again revealed unchanged appearance of rectal wall, which on sigmoidoscopy appeared normal. One year after completion of treatment, a CT/PET scan (December 2007) confirmed no residual active disease in the pelvis or elsewhere. Last CT scan from June 2009 confirmed no evidence of recurrence and the patient remains well and free of disease on clinical examination 4.5 years after completion of treatment ( 6 years after diagnosis).

\section{Discussion}

EPSCC - initially described by Duguid and Kennedy in 1930 [5] - has been increasingly recognised as a distinct entity from that of lung SCC with a different biological behaviour and prognosis. More specifically, the SCC of the gastrointestinal tract is a rare entity and typically presents at advanced stage. We present this case for its rarity and to share our positive experience in terms of outcome from treatment. Most available literature on this condition exists in the form of case reports and retrospective studies and therefore, much remains to be uncovered about the natural history and the optimal treatment of the disease.

The neuroendocrine differentiation of the tumour is usually demonstrated by immunochemical methods or electron microscopy. Positive immunoreactivity to at least 2 neuroendocrine markers is required [6], which in our case were CD56 and chromogranin. The disease staging is evaluated in a similar fashion to the one used for SCC of the lung and is essentially divided into 2 major groups: limited disease or 
extensive disease, purely based on the ability to cover the whole disease in an acceptable radiotherapy portal.

In a retrospective study of 64 cases in the United States [1] (2004), the leading location for EPSCC was colon and rectum with a median age at presentation of 55 years and slight male predominance. The median survival ranged from 6-12 months for treated patients and in weeks for untreated patients. Another retrospective study of 24 patients [7] from Korea reported a median overall survival of 15.3 months and a 3-year survival rate of $30 \%$.

The treatment approach is extrapolated from experience with SCLC. Studies up to date have invariably shown dismal prognostic outcomes for EPSCC as the disease is universally fatal and the 5 -year survival rate as low as $13 \%$. The options for extensive disease are to offer systemic chemotherapy or best supportive care. The approach for limited disease is not as clear. Some suggest only local treatments using surgery or radiotherapy and others suggest multimodality approach or chemotherapy alone [8]. One study on 54 patients with limited disease EPSCC reported an unfavourable outcome with local therapy alone (median disease-free survival of 5-6 months). In another evaluation of 81 patients with EPSCC, the majority with limited disease, the combination of chemotherapy and radiation therapy was found to be as effective as surgery [9]. The majority favour a combined modality approach using sequential or concurrent chemoradiotherapy [7].

Our treatment approach was unique, as we employed sequential chemoradiation followed by maintenance chemotherapy resulting in a long-term disease-free survival. We know from SCLC experience that these patients do respond to combined modality treatment initially but usually present with relapse fairly quickly. In the above study of 81 patients, the median time to relapse following initial response in patients with limited disease was 11.5 months [9]. Another study of 16 patients with reported rectal SCC had only 3 long-term survivors who were alive 5 years after the time of diagnosis and as expected, all of them had limited disease [10].

With our limited experience, we recommend aggressive multimodality treatment of limited-stage extrapulmonary SCLC and consideration for maintenance chemotherapy after initial good response in young and fit patients to transform initial response into a long-term disease control and possibly cure.

\section{Acknowledgements}

The authors wish to thank the following persons for their help: Dr Peng Hui Lee, Consultant Radiologist (Broomfield Hospital, Mid Essex Hospitals NHS Trust), Dr Peter A. Davis, Consultant Histopathologist (Broomfield Hospital, Mid Essex Hospitals NHS Trust). 


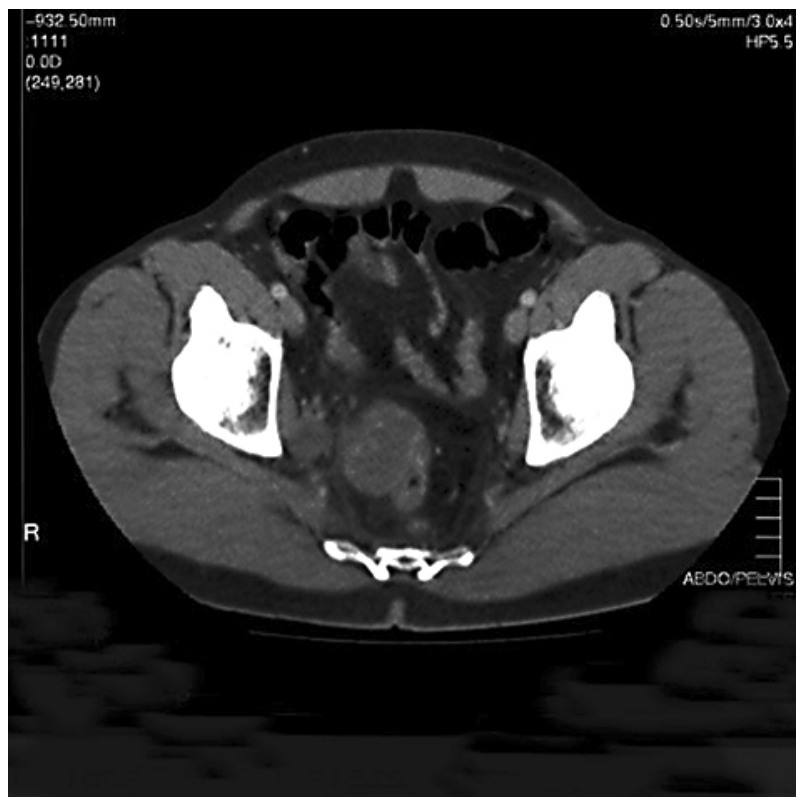

Fig. 1. CT July 2005. Rectal mass with lymph node involvement.

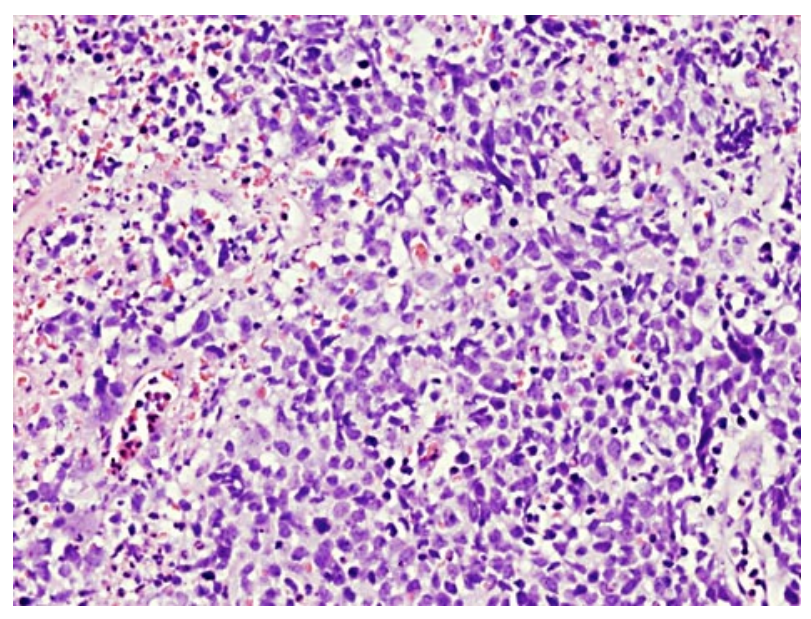

Fig. 2. The tumour with large closely packed pleomorphic nuclei and smooth hyperchromatic nuclear chromatin. A few cells show nuclear moulding, which is suggestive of neuroendocrine differentiation. As well as areas of necrosis, there are also many apoptotic cells. This, together with the high rate of mitosis, reflects the high grade nature of the tumour. 


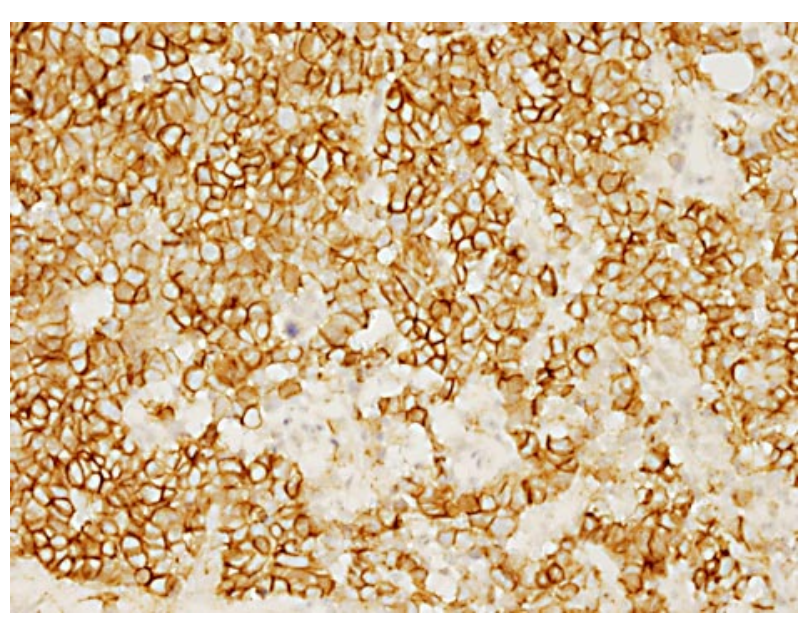

Fig. 3. Membrane staining with CD56 (NCAM - Neural Cell Adhesion Molecule), confirming the neuroendocrine nature of the tumour.

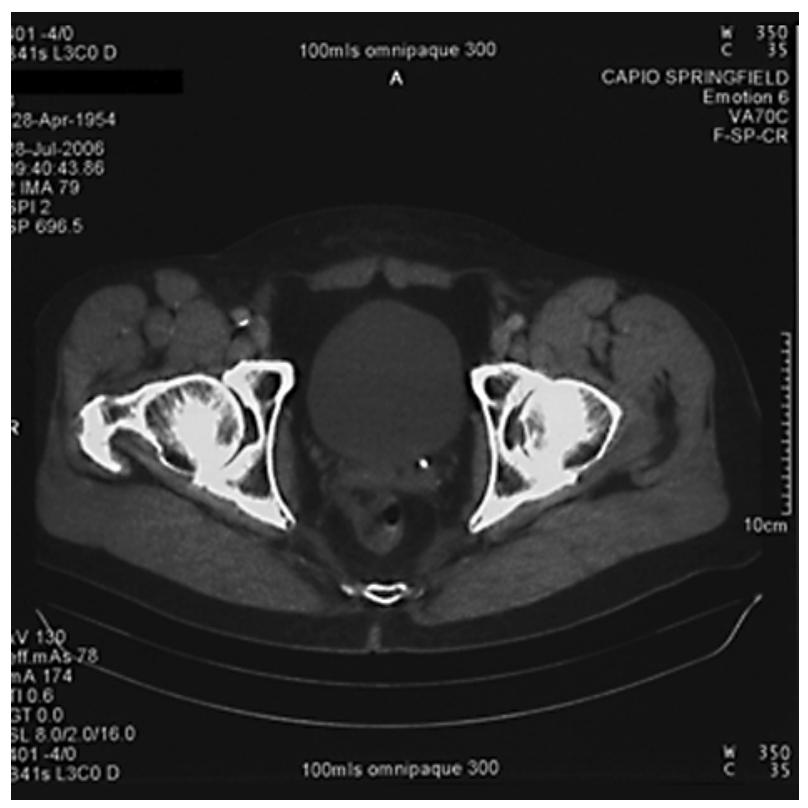

Fig. 4. CT July 2006 - post-chemoradiotherapy. Residual perirectal soft tissue at 10 o'clock at the level of seminal vesicles, extending to the mesorectal membrane which remained the same in subsequent scans and inactive in PET CT. 


\section{References}

$\checkmark 1$ Brenner B, Shah MA, Gonen M, Klimstra DS, Shia J, Kelsen DP: Small-cell carcinoma of the gastrointestinal tract: a retrospective study of 64 cases. Br J Cancer 2004;90:1720-1726.

2 Pearse AG: The diffuse neuroendocrine system and the APUN concept: related 'endocrine' peptides in brain, intestine, pituitary and anuran cutaneous glands. Med Biol 1077;55:115-125.

-3 Clery AP, Docktery MD, Waugh JM: Small cell carcinoma of the colon and rectum: a clinopathologic study. Arch Surg 1961;83:164-171.

4 Saclarides TJ, Szeluga D, Staren ED: Neuroendocrine cancers of the colon and rectum. Results of a ten-year experience. Dis Colon Rectum 1994;37:635-642.

5 Duguid J, Kennedy A: Oat cell tumors of mediastinal glands. J Pathol Bacteriol 1930;33:93-99.

6 Cebrian J, Larach SW, Ferrara A, Williamson PR, Trevisani MF, Lugan HJ, Kassir A: Small cell carcinoma of the rectum: report of two cases. Dis Colon Rectum 1999;42:274-277.

-7 Kim JH, Lee S-H, Park J, Kim HY, Lee S, Nam EM, Park J-O, Kim K, Jung CW, Im Y-H, Kang WK, Lee MH, Park K: Extrapulmonary Small-cell carcinoma: a single-institution experience. Jpn J Clin Oncol 2004;34:250254.

8 Arai K, Matsuda M: Gastric small cell carcinoma in Japan, a case report and review of the literature. Am J Clin Oncol 1998;21:458-461.

-9 Galanis E, Frytak S, Lloyd RV: Extrapulmonary small cell carcinoma. Cancer 1997;79:1729-1736.

10 Yasui O, Tsukamoto F, Kudo K: Small cell undifferentiated carcinoma of the ascending colon with rapid enlargement after resection: report of a case and review of the literature. Tohoku J Exp Med 2006;209:361-367. 